\title{
Fluctuation in straylight measurements during the visual recovery phase after small incision lenticule extraction
}

\author{
Kelvin H. Wan $\mathbb{D}^{1} \cdot$ Rachel W. Y. Tsui ${ }^{2}$ Tommy C. Y. Chan $\mathbb{D}^{1,3}$
}

Received: 27 December 2018 / Revised: 11 May 2019 / Accepted: 14 June 2019 / Published online: 9 August 2019

(c) The Author(s), under exclusive licence to The Royal College of Ophthalmologists 2019

\begin{abstract}
Purpose To investigate the postoperative straylight changes during the visual recovery phase after small incision lenticule extraction (SMILE) and their association.

Methods Seventy consecutive eyes from 37 patients with a mean age of $30.92 \pm 7.26$ years and a mean preoperative spherical equivalent of $-5.24 \pm 1.90$ dioptres undergoing myopic or myopic astigmatism SMILE correction were included in this prospective study. Patients were followed up at days 1, 3, 7, 14, 21 and 28 after standard SMILE. Straylight was measured using the C-Quant straylight meter (Oculus $\mathrm{GmbH}$, Germany) preoperatively and at each postoperative visit.

Results Preoperatively, the mean straylight measurement was $1.16 \pm 0.16$. After SMILE, the mean straylight values were $1.12 \pm 0.14$ and $1.13 \pm 0.13$ at days 7 and 14 , which were significantly reduced compared to preoperative values $(p \leq 0.028)$. Straylight returned to baseline by week $3(p=0.160)$ and remained stable onwards $(p=0.651)$. A lower ablation ratio was associated with less straylight level at days $1,3,14$ and $21(p \leq 0.0497)$ in the multivariable regression model. Likewise, better visual acuity was associated with lower straylight at days 7, 14 and 28 postoperatively $(p \leq 0.038)$. A small proportion of eyes (range: $0-12.86 \%$ ) had $\geq 0.30 \log (\mathrm{s})$ increase in postoperative straylight within the first month after SMILE.

Conclusions SMILE induced a temporary decrease in straylight. It gradually returned to the preoperative level, which could be related to a number of dynamic processes during corneal healing. In the small proportion of patients with an increase in straylight postoperatively, this can affect their visual recovery during the early postoperative period.
\end{abstract}

\section{Introduction}

Despite achieving a visual acuity of 20/20 after refractive surgery, patient satisfaction is not guaranteed because contrast sensitivity, aberration and scattering can all affect retinal image quality [1-3]. The effect of glare sensitivity resulting in visual impairment is called disability glare, which is the result of straylight $[4,5]$. Forward scattering produces straylight, which represents the light that enters the eye but does not reach the retina in a focused manner by forming a veil of light scattered over the retina. Straylight

Tommy C. Y. Chan

tommychan.me@gmail.com

1 Department of Ophthalmology and Visual Sciences, The Chinese University of Hong Kong, Hong Kong, Hong Kong

2 Hong Kong Eye Hospital, Kowloon, Hong Kong

3 Department of Ophthalmology, Hong Kong Sanatorium and Hospital, Happy Valley, Hong Kong can affect retinal sensitivity to a larger extent than classic parameters of visual function: visual acuity and contrast sensitivity [6]. Studies have suggested that straylight measurement is complementary to visual acuity to better quantify visual acuity impairment and can be considered as an ocular fitness criterion in demanding professions [7, 8].

Small incision lenticule extraction (SMILE) has gained popularity as an option to correct myopia and myopic astigmatism in recent years. The factors influencing straylight have not been thoroughly studied in SMILE. The corneal maintains its transparency due to the organized collagen fibre lattice arrangement to compensate for light scattering. After laser refractive surgery, changes in the corneal fibrils alignment can affect the optical clarity, inducing an increase in scattering postoperatively [9]. The femtosecond laser photodisruption in SMILE rather than excimer laser photoablation may elicit less cytokine release with less inflammatory reaction and corneal wound healing [10]. Yet in terms of interface reflectivity, numerous reflective particles are seen in the interface with moderate light scattering on confocal microscopy after SMILE [11]. 
The early recovery of visual function after SMILE continues to be debated; studies have reported that the visual recovery was slightly slower in SMILE in the very early postoperative period $[12,13]$. Patients after SMILE also experienced more visual fluctuation and episodic blurring than those after laser in situ keratomileusis (LASIK) in the early postoperative period [14].

Patients pay a great deal of attention to their visual recovery after refractive surgery because nearly all of them have a corrected distance visual acuity (CDVA) of 20/20 or better preoperatively. Patient-reported quality of vision was reported to be poorer 1 week following SMILE than LASIK [15]. We would expect that the extraction of the lenticule, which creates an intrastromal pocket, would inevitably disturb the lattice arrangement of the fibres, resulting in elevated levels of forward scattering. As patient's expectation has increased over time, one challenge is to evaluate factors other than visual acuity, such as straylight, that can affect visual quality. It is important to evaluate how straylight would fluctuate during the visual recovery period and to identify the factors associated with straylight. Despite providing excellent efficacy and safety for myopic and myopic correction corrections, the early recovery of visual function after SMILE is not well described. To address these issues, our study aims to analyse the straylight changes and their associations during this very early postoperative period by a prospective design to evaluate the outcomes of SMILE at days 1, 3, 7, 14, 21 and 28 after surgery.

\section{Subjects and methods}

\section{Patients}

This prospective case series included consecutive patients undergoing SMILE for myopic or myopic astigmatism correction with a target of plano between June 2017 to September 2017 at the University Eye Center, the Chinese University of Hong Kong. Preoperative evaluation included uncorrected distance visual acuity (UDVA), CDVA, manifest and cycloplegic refraction, intraocular pressure, slitlamp and fundal examinations, corneal topography using Pentacam HR (Oculus GmbH, Germany) and straylight measurement (described below). Inclusion criteria included CDVA of 20/20 or better with stable refractions for at least 1 year before surgery and an absence of other ocular or relevant systemic diseases. Eyes undergoing SMILE monovision in presbyopia patients were excluded. Patients were asked to withhold contact lens wear ( 2 weeks for soft contact lens and 1 month for hard contact lens) prior to preoperative evaluation. Written informed consent was obtained; the study was approved by the Joint Chinese
University of Hong Kong-New Territories East Cluster Clinical Research Ethics Committee and adhered to the tenets of the Declaration of Helsinki.

\section{Straylight measurement}

Straylight was quantified using the C-Quant straylight meter (Oculus $\mathrm{GmbH}$, Germany), which is based on the compensation comparison method. In brief, the meter compares the intensity of a counterphase flickering that is required to compensate an induced flickering, which is considered a proxy of forward scattering [16]. The results were recorded on a logarithmic scale as $\log (\mathrm{s})$. All measurements had an estimated standard deviation and shape factor $(Q)<0.08$ and $>1.00$, respectively, which were considered reliable by the instrument [17]. Measurements were performed under low mesopic conditions by the same technician.

\section{Surgical technique}

All SMILE procedures were performed using a $500-\mathrm{kHz}$ Visumax femtosecond laser platform (Carl Zeiss Medite, Germany) with pulse energy between 130 and $140 \mathrm{~nJ}$ by the same surgeon (TC). The intended cap thickness was 120 to $140 \mu \mathrm{m}$ with an intended diameter of $7.5 \mathrm{~mm}$, while the diameter of the lenticule was $6.5 \mathrm{~mm}$ with a transition zone of $0.1 \mathrm{~mm}$. A single side cut of $3 \mathrm{~mm}$ circumferential length was created in the superior position. The lenticule was dissected and separated through the side incision and manually removed. The corneal interface was then flushed with balanced salt solution (BSS). All patients received topical levofloxacin $0.5 \%$ and prednisolone acetate $1 \%$ ophthalmic suspension four times a day for 1 week postoperatively. Preservative-free artificial teardrops were continued for 3 months postoperatively.

\section{Statistical analysis}

Statistical analyses were performed using Stata, version 14.0 (StataCorp). We compared the preoperative and postoperative straylight values using a linear mixed-effect model fitted with $\log (\mathrm{s})$ as a response over time. We used random intercepts to account for the repeated measurements over time, with eyes nested within the subject to account for the fact that eyes from the same individual are more likely to have similar measurements. A univariable linear regression model was built using the postoperative straylight measurements as the dependent variable, a number of procedure-related parameters and visual acuity were used as the independent variables. Variables with statistically significant association were then used to build the multivariable linear regression model. The regression models accounted for multiple measurements per patient by 
calculating the standard errors clustered on the patient. $P<$ 0.05 (two-tailed) was considered significant.

\section{Results}

Seventy eyes of 37 patients undergoing SMILE (11 males, 26 females) were included. The mean age of the patients was $30.92 \pm 7.26$ years, the mean spherical refraction was $-4.88 \pm 1.68$ dioptres (D) and mean cylindrical refraction was $-1.04 \pm 0.86 \mathrm{D}$. The preoperative mean keratometry and central corneal thickness were $43.71 \pm 1.22 \mathrm{D}$ and $562.13 \pm 34.96 \mu \mathrm{m}$, respectively. The mean straylight value was $1.16 \pm 0.16$ preoperatively. The straylight measurements remained stable on day $1(1.19 \pm 0.19)$ and day 3 $(1.17 \pm 0.16)$ postoperatively $(p \leq 0.338)$. After SMILE, it decreased to $1.12 \pm 0.14$ at week $1(p=0.017)$ and $1.13 \pm$ 0.13 at week $2(p=0.028)$ postoperatively. By week 3 , the straylight measurement raised to $1.196 \pm 0.18$ and then peaked at $1.204 \pm 0.17$ at week 4 ; both were increased compared to week 1 and week 2 ( $p<0.001$ for all). These measurements at weeks 3 and 4 were comparable ( $p=$ $0.651)$ and neither measurement was significantly different from baseline ( $p=0.160$ and 0.058 , respectively). Figure 1 shows the straylight measurements before and after SMILE. No complications were observed during the follow-up period, and all patients had a normal slit-lamp examination. Figure $2 \mathrm{a}-\mathrm{c}$ illustrates the UDVA, CDVA and spherical equivalent from day 1 to 1 month after SMILE. A difference of 0.3 in $\log (\mathrm{s})$ corresponds to a difference in straylight intensity of a factor of 2 [18]. Thus, a 0.3 unit increase in $\log (\mathrm{s})$ is indicative of an increase in straylight. The proportion of eyes with more than 0.3 unit increase in $\log (\mathrm{s})$ decreased from $5.71 \%$ at day 1 postoperatively to $0 \%$ at day 7 ; the proportion reached a maximum at 1 month postoperatively with $12.86 \%$ (Fig. 2d). Less than half of the

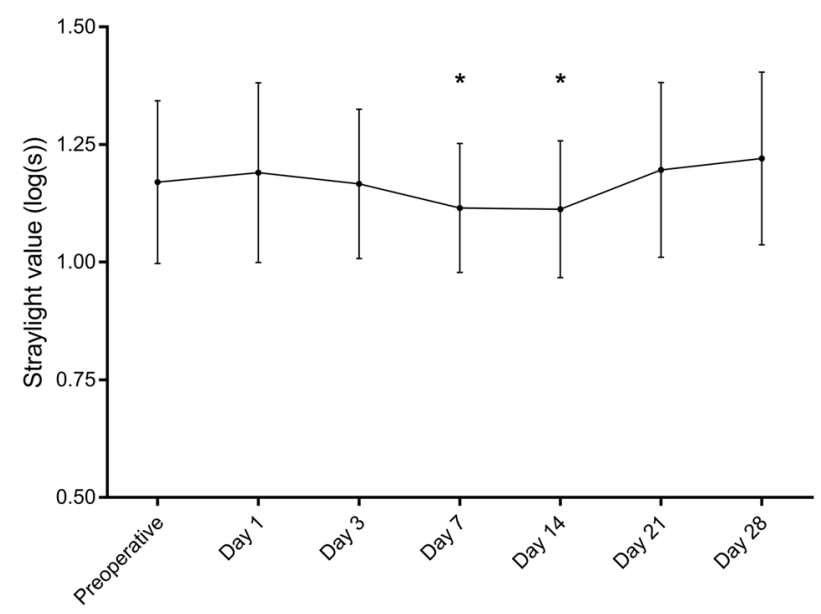

Fig. 1 Time course of straylight. * indicates significant difference $(p<0.05)$ compared to the preoperative value eyes had more than 0.05 unit increase in $\log (\mathrm{s})$ at any time after SMILE (range 20.00-37.14\%).

Table 1 shows the univariable regression model between the postoperative straylight values and SMILErelated parameters (cap thickness, ablation ratio, defined as the ratio between the lenticule thickness and preoperative central corneal thickness), preoperative straylight measurement or visual acuity. A smaller ablation ratio was associated with less postoperative straylight measurement at the majority of follow-up visits (days 1, 3, 14 and 21, $p \leq 0.0497$ ). A better UDVA or CDVA was associated with lower postoperative straylight level at most of the time points. No association was demonstrated between the postoperative straylight values and the cap thickness or the preoperative straylight level (except for day 3 postoperatively in the latter). In the multivariable regression model, after adjusting for the other variables, the ablation ratio remained significantly associated with the postoperative straylight measurements (Table 2). When UDVA was used in the multivariable regression model instead of CDVA (if both were significant in the univariable regression model), the results remained unchanged (data not shown).

\section{Discussion}

Visual perception is different from visual acuity; quality of vision is not limited to visual acuity but other effects such as straylight must be considered. Intraocular straylight is caused by light scattered towards the retina (forward scatter), the phenomenon is complex and occurs when light passing through the ocular medium deviates from its original trajectory due to the inhomogeneity along its path. Unlike backward scattering, forward scattering reaches the retina; this scattering results in a veil of light known as straylight, its influence on the retinal image is equated to a superimposed veiling luminance [19]. Straylight is equivalent to disability glare as defined by the Commission Internationale de l'Eclairage and is expressed by its equivalent luminance as the ratio of light scattered towards the retina at a certain angular distance and the total amount of light entering the eye [5]. The amount of straylight is quantified $\operatorname{logarithmically}$ as $\log (\mathrm{s})$ and the effect on visual performance by an increase of $0.1 \log (\mathrm{s})$ is comparable to a 1 line loss of visual acuity on the logMAR scale [20]. Noncataractous straylight values increases with age as: $\log (\mathrm{s})=$ constant $+\log (1+($ age/65) [4] as depicted in Fig. 3 [21]. The lens is the dominant factor accounting for the increase in straylight with age, even in clearest old lens [22], whereas corneal light scattering is constant with age [23]. Straylight can lead to patient's dissatisfaction after refractive surgery. These deleterious visual effects include night 


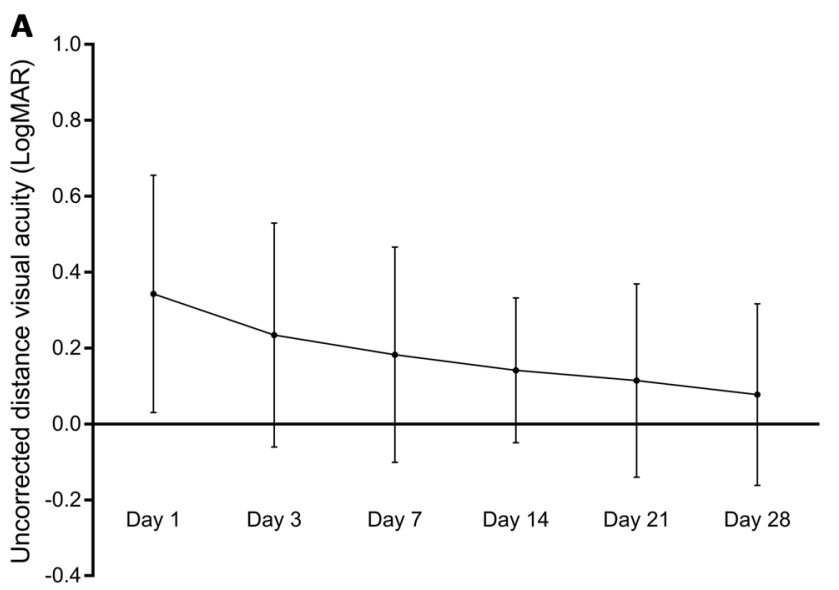

B
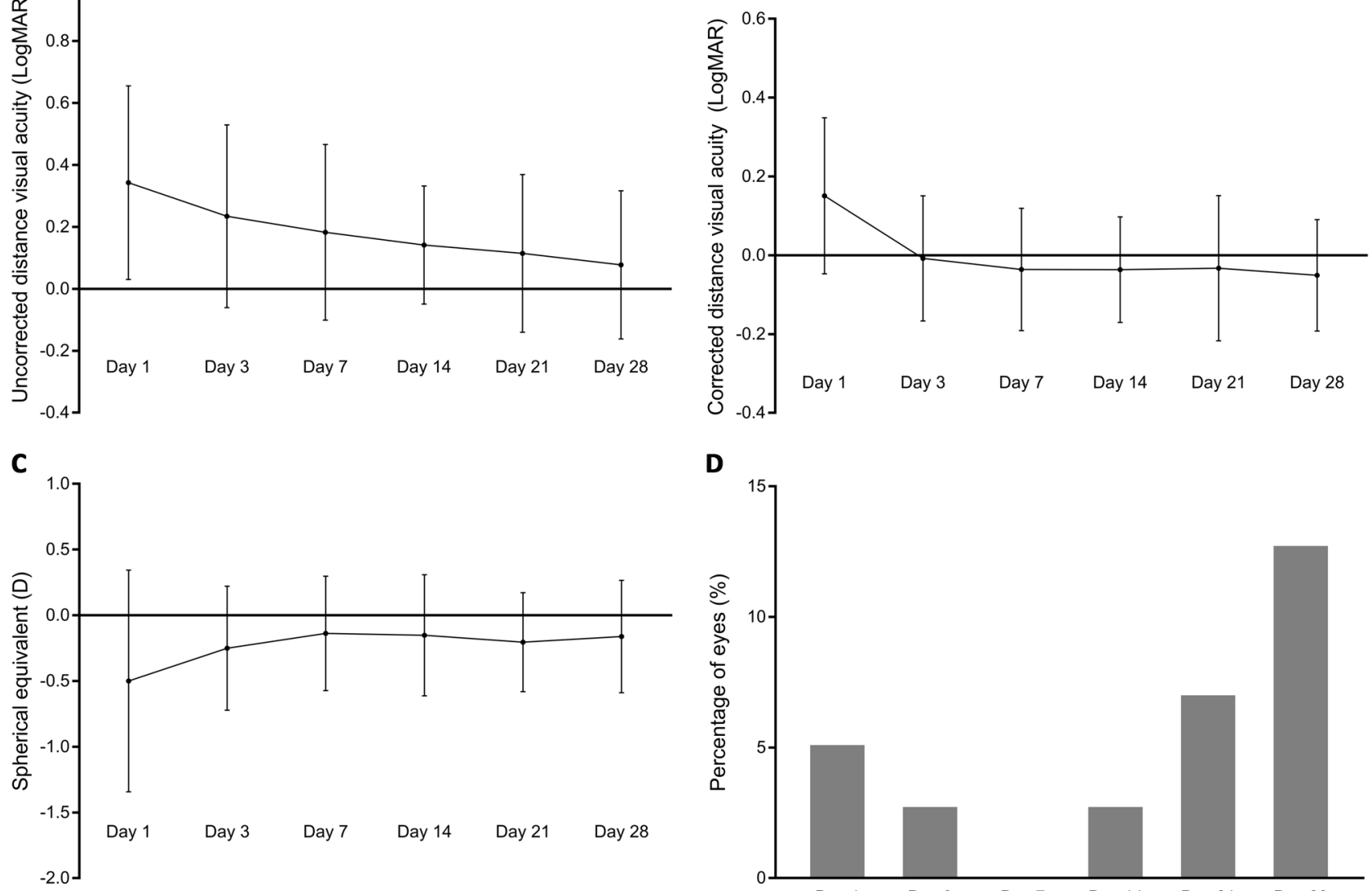

D

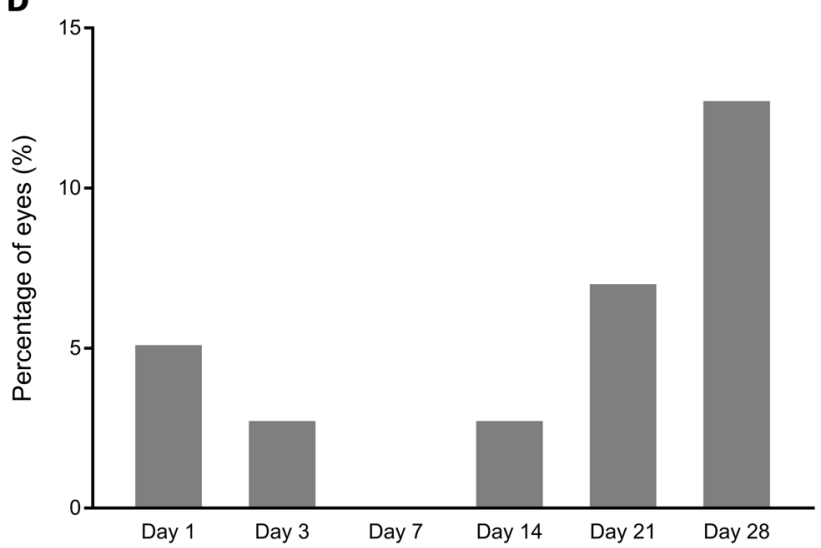

Fig. 2 Time course of a uncorrected distance visual acuity, $\mathbf{b}$ corrected distance visual acuity, $\mathbf{c}$ spherical equivalent and $\mathbf{d}$ proportion of eyes with a straylight increase of $>0.30 \log (\mathrm{s})$ after SMILE

vision disturbances, glare sensitivity, irritability to sunlight, facial recognition problem, foggy vision, reduced in colour and contrast sensitivity and so on [5, 24]. Since light scattering causes contrast loss in the retinal image, understanding forward scattering becomes important after refractive surgery.

Assessing optical quality after refractive surgery is essential and correlates with patient's satisfaction. Visual recovery following SMILE was delayed compared with the best results of modern refractive surgery $[12,13]$. In addition to higher-order aberrations, forward scattering also independently affects the retinal image quality [6]. We found higher postoperative straylight was associated with a larger ablation ratio, signifying a higher refractive correction and/or thinner preoperative corneal pachymetry; this suggests a dose-response relationship between the amount of postoperative scattering and the proportion of removed corneal tissue. Since the density of the cornea decreases progressively towards the deeper layer of the stroma [25], photodisruption becomes more irregular, giving rise to a higher straylight value in eyes with larger ablation ratio. Using double-pass aberrometry, several studies also reported that the increase in postoperative objective scatter index (OSI) was correlated with the preoperative spherical equivalent [26, 27].

Straylight measurement using the C-Quant either decreased or remained stable after corneal refractive surgery [28-32]. Our findings agree with a previous study that reported no significant increase in forward scattering after SMILE in 1 month; [28] however, unlike the present study, it did not report on the straylight observations within the first month. Our study monitored the changes in forward scattering with frequent follow-up during the first postoperative month to better understand its impact on visual quality. Chiche et al. [15] found that the OSI was independently correlated with the UDVA in the SMILE group, but not in the LASIK group, across all postoperative timepoints, whereas Gyldenkerne et al. [26] failed to show a statistically significant association between UDVA and OSI following SMILE, which they attributed to the fluctuation of an unstable tear film. We found that better UDVA and CDVA was associated with lower postoperative straylight measured using the C-Quant straylight meter for more than half of the observation; this signifies the importance of 


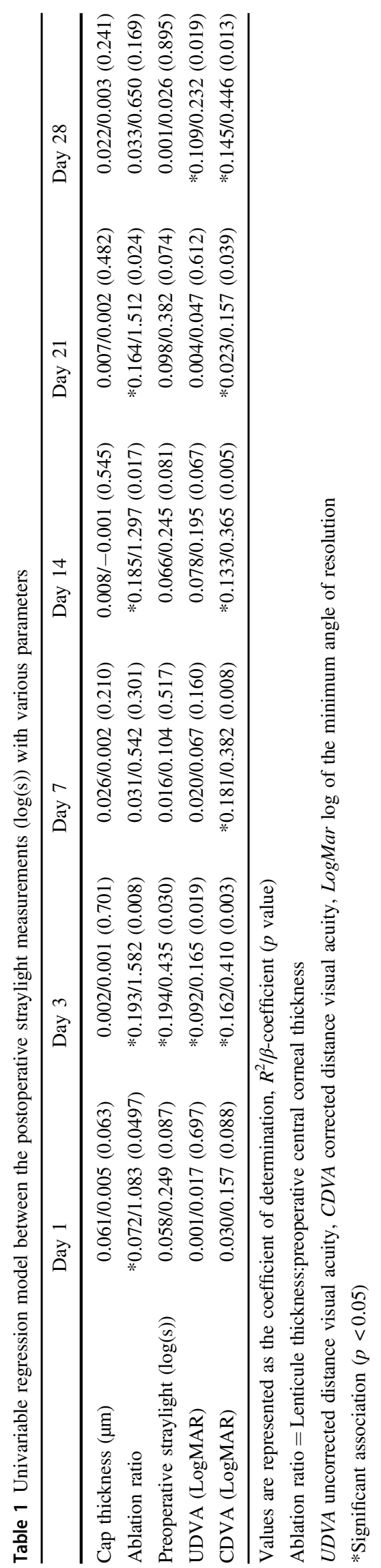

straylight changes on the visual function during the very early postoperative period. Contrary to our hypothesis, the mean straylight level did not increase compared to the baseline at any time within 1 month after SMILE; instead, it was lower than the preoperative level at weeks 1 and 2 after surgery. A similar trend was previously reported in a study which found that straylight was reduced 15 days after LASIK and returned to preoperative levels by 6 months [33]. The measurable decrease in straylight could be cancelled out by the effects of minor surface irregularity or the microdistortions created during the learning curve phase in SMILE [34], which could increase the straylight.

We hypothesize that the interplay between a number of dynamic factors could explain the fluctuation of straylight observed during the early postoperative phase following SMILE: (1) neutralization of high preoperative straylight, (2) cap-stroma interface coupling, (3) absorption of intrastromal pocket fluid, (4) Bowman's layer microdistortion and corneal lamellar wound healing. LapidGortzak et al. [29] reported a reduction in straylight values 3 months postoperatively after myopic LASIK and laserassisted subepithelial keratectomy (LASEK), whereas these measurements remained stable after hyperopic correction [32]. Since one-third of all straylight is derived from the cornea [35], the authors hypothesized that removing a part of the cornea would reduce the straylight due to its substantial contribution. They further attribute their observation to the workload of the corneal endothelium, where the residual corneal stroma that otherwise must be kept dehydrated would become less hydrated after refractive surgery, hence decreasing the straylight. To account for the difference in myopic and hyperopic correction, they speculated that the more peripheral tissue in hyperopic correction contributes less to straylight changes since the central cornea over the pupillary margin contributes most to straylight from the corneal sources [36]. Contact lens wear can increase the straylight level even after they have not been in use for some time [37]. The preoperative straylight level of $1.16 \pm 0.16$ in our study was indeed higher than the general population in other studies ranging from 0.870 to 0.931 [21, 38]. Therefore, the reduction in straylight observed in the first 2 weeks postoperatively may suggest that straylight is neutralized by SMILE. Rozema et al. [30] reported that after LASEK, the straylight level was reduced 6 months after the procedure and was correlated with the preoperative level. Similarly, they postulated that LASEK might neutralize the preoperative straylight. In the current study, we only found a significant association between the preoperative straylight level and the straylight level on day 3 postoperatively.

After the myopic correction in SMILE, the posterior surface of the cap no longer matches the underlying residual stromal bed. The residual stromal bed becomes flatter 
Table 2 Multivariable regression model between the postoperative straylight measurements $(\log (\mathrm{s}))$ with various parameters

\begin{tabular}{lllllll}
\hline & Day 1 & Day 3 & Day 7 & Day 14 & Day 21 & Day 28 \\
\hline Ablation ratio & $* 1.083(0.0497)$ & $* 1.207(0.013)$ & $/$ & $* 1.052(0.034)$ & $* 1.460(0.031)$ & $/$ \\
Preoperative straylight $(\log (\mathrm{s}))$ & $/$ & $* 0.379(0.036)$ & $/$ & $/$ & $/$ & $/$ \\
CDVA (LogMAR) & $/$ & $0.139(0.181)$ & $* 0.382(0.008)$ & $* 0.253(0.038)$ & $0.060(0.362)$ & $* 0.446(0.013)$ \\
\hline
\end{tabular}

Values are represented as $\beta$-coefficient ( $p$ value)

Ablation ratio $=$ Lenticule thickness:preoperative central corneal thickness

CDVA corrected distance visual acuity, LogMar log of the minimum angle of resolution

/ indicates the variable was not used in the regression model because it was not significant $(p \geq 0.05)$ in the univariable model

* indicates significant association $(p<0.05)$

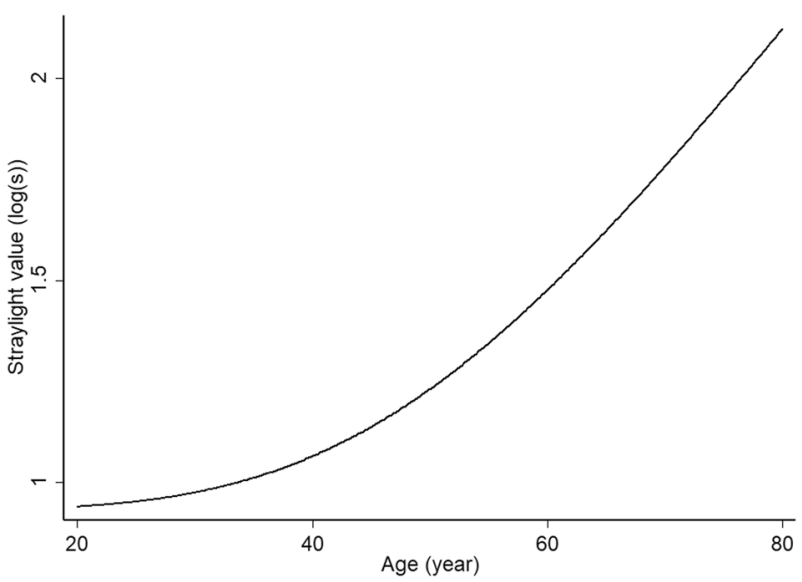

Fig. 3 Graph showing straylight values as a function of age based on the van den Berg age reference of $\log (\mathrm{s})=0.931+\log (1+$ (age/65) [4]

than the posterior surface of the cap, which follows the curvature of the anterior corneal surface. The cap becomes too large because, for the same chord length, the steeper surface has a larger area. During wound healing, the quasispherical posterior cap surface must change its shape to conform to the underlying stroma to match the larger area of the posterior surface of the cap to the smaller area of the residual stromal bed. This process involves compression of the corneal cap with possible segmental shrinking or by overlapping the cap with the residual stroma. Astigmatism correction further complicates the situation because the lenticule is thicker in the meridian perpendicular to the astigmatism axis. Theoretical modelling in LASIK revealed that the mismatch between the apposition of the LASIK flap and the residual stroma after ablation increases with the magnitude of the attempted refractive correction [39]. This interface mismatch would be higher in high myopic eyes with the steep corneal surface. This could account for the significant association between the ablation ratio and the straylight up to 3 weeks postoperatively demonstrated in our study. We speculate that over time, the apposition becomes more regular and the cap can better adhere to the stroma, which in turn improves the congruity and reduces forward scattering.

Most surgeons, including the authors, flush the intrastromal pocket with BSS or saline. This is under the presumptive belief that flushing can reduce the inflammatory cytokines generated from the photodisruption/surgical manipulation, contaminants and epithelial cells that could lead to epithelial ingrowth. Histological analysis of rabbit eyes at $24 \mathrm{~h}$ after SMILE found that in the eyes with irrigation with BSS, there were undulated but undisrupted stromal collagen bundles in the anterior and posterior stroma compared to those without irrigation. Furthermore, the majority of the cornea in the irrigation group had small pockets with fluid retention along the extracted lenticule plane [40]. The resorption of this fluid layer over time could reflect the reduction of forward scattering within the first 2 weeks postoperatively in our study.

Bowman's layer microdistortions, detected by optical coherence tomography (OCT), were observed following SMILE with greater numbers in the high myopia group [34, 41, 42]. These Bowman's layer microdistortions are thought to originate from remodelling of the mismatched cap-stroma interface. Luo et al. [42] observed that the number of microdistortions remained the same after 1 month, thus they hypothesized that this phenomenon is not caused by tissue oedema in the early stage after SMILE. By quantitatively mapping these microdistortions as an index using OCT, Shetty et al. [43] showed that these microdistortions returned to the preoperative levels by 3 months postoperatively. Haze formation and anterior keratocyte loss have been implicated as the main culprit of scattering after excimer laser surgery [44]. Confocal microscopy revealed a network of activated keratocytes and increased reflectivity from the extracellular matrix after SMILE [45], and extracellular matrix reformation was reported to peak at 1 month postoperatively [9]. Mastropasqua et al. [11] postulated that the increased reflectivity was attributed by two different elements: the activated 
keratocytes and the reflective particles in the extracellular matrix. The reflective active keratocytes were believed to be related to the tissue inflammatory response, whereas the reflective particles could represent the residual organic cellular constituents subsequent to the femtosecond laser photodisruption action. Scanning electronic microscopy of the human donor cornea lenticule bed revealed that the surface texture of the stromal lenticule bed following SMILE had a more irregular appearance with more fringed collagen lamellae when compared to stromal beds after FSLASIK; the authors hypothesized that these concavities represent the site of the cavitation gas bubble formation from the photodisruption [46]. Thus, the increase in the forward scattering after 2 weeks postoperatively in the current study could be associated with the corneal lamellar wound healing process, which continues to occur after 2 weeks and peaked at 1 month.

Corneal wound healing is a complex cascade involving different cytokines and growth factors; they undermine the predictability and stability of refractive surgeries and account for the discrepancies between the attempted and achieved visual outcomes [47]. A number of dynamic processes occur during the early postoperative period; the interplay between these mechanisms and their relative contribution during the different phases of early recovery could account for the fluctuation in forward scattering. Our study is limited by the small sample size; more observations might have increased the power of the linear regression model. Further studies using confocal microscopy, OCT and histological examination may help better our understanding of these factors contributing to straylight changes after SMILE.

Straylight is an essential functional parameter to consider in evaluating the outcomes after SMILE. Our findings indicate an initial drop in straylight following SMILE followed by a gradual return to preoperative values from 3 weeks onwards. A lower postoperative straylight level is associated with better visual acuity and a smaller ablation ratio. A number of dynamic processes occur during the early postoperative period: cap-stroma interface coupling, absorption of intrastromal pocket fluid, Bowman's layer microdistortion and corneal remodelling. The interplay between these mechanisms and their relative contribution during the different phases of recovery accounts for the fluctuation in forward scattering. Further studies are needed to investigate the aetiologies accounting for these fluctuations in straylight during the very early postoperative period.

\section{Summary}

\section{What was known before}

- Forward scattering independently affects the retinal image quality and correlates with patient's satisfaction.
- Visual recovery following SMILE was delayed compared to other corneal refractive surgeries.

\section{What this study adds}

- An initial drop in straylight following SMILE occurred at 1 and 2 weeks postoperatively, followed by a return to preoperative values at 1 month.

- Lower postoperative straylight level is associated with better visual acuity and a smaller ablation ratio.

\section{Compliance with ethical standards}

Conflict of interest The authors declare that they have no conflict of interest.

Publisher's note: Springer Nature remains neutral with regard to jurisdictional claims in published maps and institutional affiliations.

\section{References}

1. Bailey MD, Mitchell GL, Dhaliwal DK, Boxer Wachler BS, Zadnik K. Patient satisfaction and visual symptoms after laser in situ keratomileusis. Ophthalmology. 2003;110:1371-8.

2. Krueger RR, Thornton IL, Xu M, Bor Z, van den Berg TJ. Rainbow glare as an optical side effect of IntraLASIK. Ophthalmology. 2008;115:1187-95 e1181.

3. Schallhorn SC, Tanzer DJ, Kaupp SE, Brown M, Malady SE. Comparison of night driving performance after wavefront-guided and conventional LASIK for moderate myopia. Ophthalmology. 2009;116:702-9.

4. van den Berg TJ. On the relation between glare and straylight. Doc Ophthalmol. 1991;78:177-81.

5. Vos JJ. Disability glare-a state of the art report. CIE J. 1984; 3:39-53.

6. van den Berg TJTP, van Rijn LJ, Kaper-Bongers R, Vonhoff DJ, Völker-Dieben HJ, Grabner G, et al. Disability glare in the aging eye. Assessment and impact on driving. J Optom. 2009; 2:112-8.

7. Bal T, Coeckelbergh T, Van Looveren J, Rozema JJ, Tassignon MJ. Influence of cataract morphology on straylight and contrast sensitivity and its relevance to fitness to drive. Ophthalmologica. 2011;225:105-11.

8. van Bree MC, van Verre HP, Devreese MT, Larminier F, van den Berg TJ. Straylight values after refractive surgery: screening for ocular fitness in demanding professions. Ophthalmology. 2011;118:945-53.

9. Chang SW, Benson A, Azar DT. Corneal light scattering with stromal reformation after laser in situ keratomileusis and photorefractive keratectomy. J Cataract Refract Surg. 1998;24:1064-9.

10. Dong Z, Zhou X, Wu J, Zhang Z, Li T, Zhou Z, et al. Small incision lenticule extraction (SMILE) and femtosecond laser LASIK: comparison of corneal wound healing and inflammation. Br J Ophthalmol. 2014;98:263.

11. Mastropasqua L, Calienno R, Curcio C, Mastropasqua R, Nubile $\mathrm{M}$, Salgari $\mathrm{N}$, et al. In vivo and ex vivo evaluation of inflammation and apoptosis induced after SMILE procedures for different refractive error range. Curr Eye Res. 2017;42:701-7. 
12. Shah $\mathrm{R}$, Shah $\mathrm{S}$. Effect of scanning patterns on the results of femtosecond laser lenticule extraction refractive surgery. J Cataract Refract Surg. 2011;37:1636-47.

13. Kamiya K, Igarashi A, Ishii R, Sato N, Nishimoto H, Shimizu K. Early clinical outcomes, including efficacy and endothelial cell loss, of refractive lenticule extraction using a $500 \mathrm{kHz}$ femtosecond laser to correct myopia. J Cataract Refract Surg. 2012;38:1996-2002.

14. Ang M, Ho H, Fenwick E, Lamoureux E, Htoon HM, Koh J, et al. Vision-related quality of life and visual outcomes after smallincision lenticule extraction and laser in situ keratomileusis. J Cataract Refract Surg. 2015;41:2136-44.

15. Chiche A, Trinh L, Saada O, Faure JF, Auclin F, Baudouin C, et al. Early recovery of quality of vision and optical performance after refractive surgery: small-incision lenticule extraction versus laser in situ keratomileusis. J Cataract Refract Surg. 2018;44:1073-9.

16. Franssen L, Coppens JE, van den Berg TJ. Compensation comparison method for assessment of retinal straylight. Invest Ophthalmol Vis Sci. 2006;47:768-76.

17. Coppens JE, Franssen L, van Rijn LJ, van den Berg TJ. Reliability of the compensation comparison stray-light measurement method. J Biomed Opt. 2006;11:34027.

18. Gilliland KO, Johnsen S, Metlapally S, Costello MJ, Ramamurthy $\mathrm{B}$, Krishna PV, et al. Mie light scattering calculations for an Indian age-related nuclear cataract with a high density of multilamellar bodies. Mol Vis. 2008;14:572-82.

19. Fry GA, Alpern M. The effect of a peripheral glare source upon the apparent brightness of an object. J Opt Soc Am. 1953;43:189-95.

20. van der Meulen IJ, Gjertsen J, Kruijt B, Witmer JP, Rulo A, Schlingemann RO, et al. Straylight measurements as an indication for cataract surgery. J Cataract Refract Surg. 2012;38:840-8.

21. Van Den Berg TJ, Van Rijn LJ, Michael R, Heine C, Coeckelbergh T, Nischler C, et al. Straylight effects with aging and lens extraction. Am J Ophthalmol. 2007;144:358-63.

22. van den Berg TJ. Analysis of intraocular straylight, especially in relation to age. Optom Vis Sci. 1995;72:52-9.

23. JK IJ, de Waard PW, van den Berg TJ, de Jong PT. The intraocular straylight function in 129 healthy volunteers; dependence on angle, age and pigmentation. Vis Res. 1990;30:699-707.

24. van den Berg TJ. Importance of pathological intraocular light scatter for visual disability. Doc Ophthalmol. 1986;61:327-33.

25. Randleman JB, Dawson DG, Grossniklaus HE, McCarey BE, Edelhauser HF. Depth-dependent cohesive tensile strength in human donor corneas: implications for refractive surgery. J Refract Surg. 2008;24:S85-89.

26. Gyldenkerne A, Ivarsen A, Hjortdal J. Optical and visual quality after small-incision lenticule extraction. J Cataract Refract Surg. 2019;45:54-61.

27. Donate D, Thaëron R. SMILE with low energy levels: assessment of early visual and optical quality recovery. J Refract Surg. 2019; 35:285-93.

28. Xu L, Wang Y, Li J, Liu Y, Wu W, Zhang H, et al. Comparison of forward light scatter changes between SMILE, femtosecond laser-assisted LASIK, and epipolis LASIK: results of a 1-year prospective study. J Refract Surg. 2015;31:752-8.

29. Lapid-Gortzak R, van der Linden JW, van der Meulen I, Nieuwendaal C, van den Berg T. Straylight measurements in laser in situ keratomileusis and laser-assisted subepithelial keratectomy for myopia. J Cataract Refract Surg. 2010;36:465-71.

30. Rozema JJ, Coeckelbergh T, Van den Berg TJ, Trau R, Duchateau NC, Lemmens $\mathrm{S}$, et al. Straylight before and after LASEK in myopia: changes in retinal straylight. Invest Ophthalmol Vis Sci. 2010;51:2800-4.

31. Beerthuizen JJ, Franssen L, Landesz M, van den Berg TJ. Straylight values 1 month after laser in situ keratomileusis and photorefractive keratectomy. J Cataract Refract Surg. 2007;33:779-83.

32. Lapid-Gortzak R, van der Linden JW, van der Meulen IJ, Nieuwendaal CP, Mourits MP, van den Berg TJ. Straylight before and after hyperopic laser in situ keratomileusis or laser-assisted subepithelial keratectomy. J Cataract Refract Surg. 2010;36:1919-24.

33. Lorente-Velazquez A, Nieto-Bona A, Collar CV, Gutierrez Ortega AR. Intraocular straylight and contrast sensitivity (1/2) and 6 months after laser in situ keratomileusis. Eye Contact Lens. 2010;36:152-5.

34. Yao P, Zhao J, Li M, Shen Y, Dong Z, Zhou X. Microdistortions in Bowman's layer following femtosecond laser small incision lenticule extraction observed by Fourier-domain OCT. J Refract Surg. 2013;29:668-74.

35. de Waard PW, JK IJ, van den Berg TJ, de Jong PT. Intraocular light scattering in age-related cataracts. Invest Ophthalmol Vis Sci. 1992;33:618-25.

36. Franssen L, Tabernero J, Coppens JE, van den Berg TJ. Pupil size and retinal straylight in the normal eye. Invest Ophthalmol Vis Sci. 2007;48:2375-82.

37. van der Meulen IJ, Engelbrecht LA, van Vliet JM, Lapid-Gortzak R, Nieuwendaal CP, Mourits MP, et al. Straylight measurements in contact lens wear. Cornea. 2010;29:516-22.

38. Rozema JJ, Van den Berg TJ, Tassignon MJ. Retinal straylight as a function of age and ocular biometry in healthy eyes. Invest Ophthalmol Vis Sci. 2010;51:2795-9.

39. Charman WN. Mismatch between flap and stromal areas after laser in situ keratomileusis as source of flap striae. J Cataract Refract Surg. 2002;28:2146-52.

40. Liu YC, Jayasinghe L, Ang HP, Lwin NC, Yam GH, Mehta JS. Effect of intraoperative corneal stromal pocket irrigation in small incision lenticule extraction. Biomed Res Int. 2015;2015:928608.

41. Miao H, Liu X, Tian M, Zhao J, Fang X, Zhou X. Short-term observation of intraocular scattering and Bowman's layer microdistortions after SMILE-CCL. J Refract Surg. 2018;34:387-92.

42. Luo J, Yao P, Li M, Xu G, Zhao J, Tian M, et al. Quantitative analysis of microdistortions in Bowman's Layer using optical coherence tomography after SMILE among different myopic corrections. J Refract Surg. 2015;31:104-9.

43. Shetty R, Francis M, Shroff R, Pahuja N, Khamar P, Girrish M, et al. Corneal biomechanical changes and tissue remodeling after SMILE and LASIK. Invest Ophthalmol Vis Sci. 2017;58:5703-12.

44. Netto MV, Mohan RR, Medeiros FW, Dupps WJ Jr., Sinha S, Krueger RR, et al. Femtosecond laser and microkeratome corneal flaps: comparison of stromal wound healing and inflammation. J Refract Surg. 2007;23:667-76.

45. Agca A, Ozgurhan EB, Yildirim Y, Cankaya KI, Guleryuz NB, Alkin Z, et al. Corneal backscatter analysis by in vivo confocal microscopy: fellow eye comparison of small incision lenticule extraction and femtosecond laser-assisted LASIK. J Ophthalmol. 2014;2014:265012.

46. Luft N, Schumann RG, Dirisamer M, Kook D, Siedlecki J, Wertheimer C, et al. Wound healing, inflammation, and corneal ultrastructure after SMILE and femtosecond laser-assisted LASIK: a human ex vivo study. J Refract Surg. 2018;34:393-9.

47. Dupps WJ, Wilson SE. Biomechanics and wound healing in the cornea. Exp eye Res. 2006;83:709-20. 